\title{
Influence of trimming of resistive thick films on nonlinearity of their current vs. voltage characteristics
}

\author{
PAVEL MACH ${ }^{\prime}$, PAVEL SVASTA ${ }^{2}$ \\ 'Department of Electrotechnology, Faculty of Electrical Engineering. Czech Technical University in Prague \\ Teclnická 2, 16627 Prague 6, Czech Republic \\ PJone $(++420) 224352122$, Fax $(++420) 224353$ 949; E-mail: machc@fel.cuut.cz \\ ${ }^{2}$ University "Politehnica" Bucharest, Spl. Independentei 313, 77206 Bucharest, Romania \\ Phone +40-1-4116674, Fax +40-1-4115182; E-mail: svasta@cadtieccp.pub.ro
}

\begin{abstract}
Investigation of changes of nonlinearity of thick resistive films trimmed by laser and by grinding has been carried out. Laser trimming has been realized by an Ar laser, mechanical trimming by grinding of the layer by a milling machine using a diamond miller. The simplest method of trimming has been used - the resistors have been trimmed by realization of a grave approximately in the middle of their length perpendicularly to the axes of the resistors. The length of the trimming grave has been from $10 \%$ to $80 \%$ of the width of the resistor. All measured values of nonlinearity after trimming have been recalculated for starting values of the resistors. Nonlinearity has been examined for constant current density at the narrowest cross section of the resistive layer. It has been found that changes of nonlinearity caused by trimming depend on the ratio of the width and the length of the resistors. The changes have been very similar for resistors trimmed by the laser and for resistors trimmed mechanically.
\end{abstract}

Keywords: thick film resistors, nonlinearity of I-V characteristic, laser trimming, mechanical trimming.

\section{Introduction}

Thick film resistors are components widely used in electronic equipments. The resistors are fabricated by thick film technology on insulating substrates; usually on alumina. The resistances of the resistors after printing and curing are lower than requested values. Adjusting of the resistors to nominal values is carried out by trimming. This process is usually realized by a laser, but, in some cases, other technologies are used, e.g. abrasive trimming or a chemical way.

The laser trimming is a very fast and effective method, but the equipment is very expensive. Therefore it is used for mass production mostly. Mechanical trimming based on grinding of the part of the layer is used for the laboratory experiments usually. Other types of trimming are used in special cases only.

The main disadvantage of the laser trimming is curing of the borders of a trimming groove. A laser beam irradiates the layer. The power density of the laser beam is high. Therefore the layer is melted and evaporated at the area of irradiation. However, the power profile of the laser beam is not rectangular. Therefore the borders of the groove, which are not evaporated, are also irradiated during trimming, but the power of the beam at these places is not sufficient to evaporate the layer. These parts are partially melted or cured only. But the curing causes that the layer has other properties in comparison with the layer, which has not been irradiated (see Fig. 1).

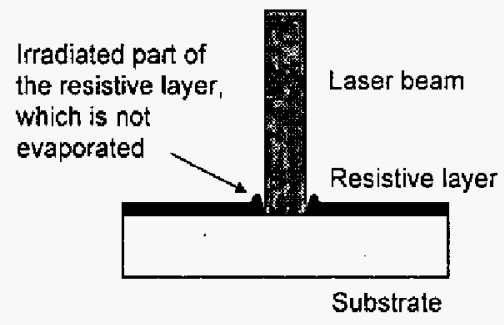

Fig. 1. The power cross section of the laser beam used for trimming is not rectangular, but it is usually similar to the Gaussian curve. Therefore borders of the trimming groove are cured, but not evaporated.

It is possible to observe that the resistive layers have lower noise and nonlinearity of the current vs. voltage characteristics before than after trimming. It is assumed that the reason of increase of these non-requested properties of the layer is curing of the borders of the trimming groove by the laser beart.

The goals of the work has been to compare changes of nonlinearity of the resistive layers caused by laser trimming and by mechanical trimming based on grinding of the layer. 


\section{Theory}

Nonlinearity of the current vs. voltage characteristic is expressed usually by Third harmonics index THI. It follows from Zhigalsky theory that THI can be calculated according to the equation

$$
T H I=20 \cdot \log \left(0,25 R_{r} \frac{L}{b \cdot h} \rho_{01}\left[\beta+\frac{A A_{v} E_{v}}{k T_{0}^{2} \rho_{0 \mathrm{at}}} \exp \left(-\frac{E_{v}}{k T_{0}}\right)\right]\right)
$$

Where $R_{T} \ldots$ thermal resistance, $\rho_{01} \ldots$ the electrical resistivity of layer upon the equilibrium temperature T $0, \beta$... TCR of the layer, $b$... the width, $L$ $\ldots$ the length, $h \ldots$ the thickness of the layer, $A \ldots$ the coefficient of proportion, $A_{v} \ldots$ the entropy multiplier, $E_{v} \ldots$ the smallest activation energy of vacancy, $\mathbf{k}$... the Boltzman constant, $K$... the dimension coefficient of the layer, which is defined by the equation

$$
K=\frac{L}{b \cdot h}
$$

The trimmed resistor is shown in Fig. 2

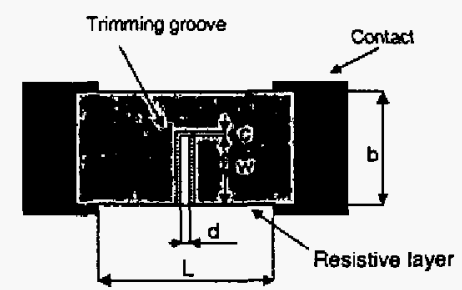

Fig. 2 Trimmed resistor

The resistance of the resistor without trimming can be expressed by the equation (3)

$$
R_{\mathrm{t}}=R_{\mathrm{is}}\left(J_{\mathrm{l}}\right) \frac{L}{b}
$$
creases

After trimming the resistance of the resistor in-

$$
\begin{aligned}
& R_{2}=R_{1 s}\left(J_{2}\right) \frac{L-d}{b}+R_{1 s}\left(J_{1}\right) \frac{d}{b-w-c}+R_{d J}\left(J_{3}\right) \frac{d+2 c}{c} \\
& J_{2}\left\langle J_{1}, \quad J_{3}\left\langle J_{1}\right.\right.
\end{aligned}
$$

Here $R_{2} \ldots$ resistance after trimming, $R_{1 s} \ldots$ resistance per square of the resistive layer, $J_{1}, J_{2}, J_{3} \ldots$ current densities, $\mathbf{R}_{\mathrm{ts}} \ldots$ resistance of the resistive layer on the border of the trimming groove, where the layer is cured by the laser beam.

The total nonlinearity of the resistor is caused by nonlinearity of the contacts, by nonlinearity of the parts of the resistive layer, which have not been trimmed, and by the trimmed part of the resistor. With respect to many different types of conductive mechanisms, which take part inside the resistive layer and on the border between resistive and conductive (contact) layer, the detail analysis of the contributions of particular components of nonlinearity is very difficult. On the other hand, it can be analyzed increase of nonlinearity caused by the trimming, because the other sources of nonlinearity have not been influenced by trimming.

It has been derived in [1] that it is advantageous to use a constant current density if nonlinearity of different layers is compared. Therefore the same current density has been used for comparison of nonlinearity of the layers before and after trimming. Because the cross section of the resistive layer differs in different places after trimming, the current density has been kept at the smallest cross section of the trimmed layer, where the width of the layer has been decreased by the trimming groove. Therefore, the current density at others places of the trimmed resistor have been lower.

It follows from the basic equation of nonlinearity of the current vs. voltage characteristics that the nonlinearity is the higher the higher is the current flowing through the specimen [2]. Therefore, if the contribution of nonlinearity of the contacts will be skipped in following analysis (the contacts cause significant part of nonlinearity, but its value is not changed by trimming), it is possible to state, that according to the equation (3) the resultant nonlinearity of the resistor after trimming consists of three parts: the first part is nonlinearity of the basic layer at the current density lower than that used for measurement of the non-trimmed resistor, the second is nonlinearity of strictured cross section at the area of the trimming groove, and the third part is nonlinearity of the border of the trimming groove.

The first part causes lower voltage of third harmonic frequency in comparison with the same part of non-trimmed resistor, because the current density in this part is lower than the density used by the measurement of the resistor without trimming.

The second part causes the same nonlinearity like the same part of the non-trimmed resistor, because it is flowed by the same current.

The nonlinearity caused by the third part depends on properties of the border of the trimming groove. If the resistivity of this part is higher than 
resistivity of the basic layer, the current density $J_{3}$ would be lower than $J_{1}$ and vice versa.

It follows from this analysis: when the measurement is caries out under the conditions mentioned above and total nonlinearity of the trimmed resistor is higher in comparison with nonlinearity of the resistor without trimming, the increase of nonlinearity has to be caused by the border of the trimming groove.

\section{Experimental}

The resistors have been fabricated of resistive pastes HS 8031 and FK 9231 E8. Both the pastes are on the basis of RuO, the resistance per square both the pastes has been $1 \mathrm{k} \Omega$. The contacts have been fabricated of $\mathrm{Pd} / \mathrm{Ag}$ paste. Thickness of the films has been $25 \mu \mathrm{m}$, curing temperature has been $850^{\circ} \mathrm{C}$.

The paste HS 8031 has been applied on the alumina substrates $99.5 \%$, the paste FK 9231 E8 on the AlN substrates. The substrates differ especially by thermal conductivity, alumina has the conductivity $25 \mathrm{~W} / \mathrm{mK}$, AlN $170 \mathrm{~W} / \mathrm{mK}$. The specimen is shown in Fig. 3

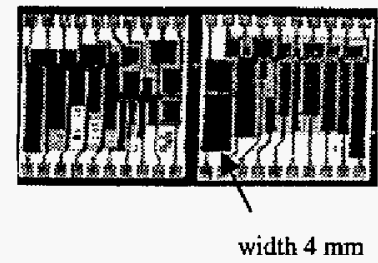

Fig. 3 Specimen with the thick film resistors

The methods of the measurement of nonlinearity have been mentioned elsewhere [3]. The index nonlinearity has been calculated according to the formula

$$
T H I=\frac{U_{3}}{U_{1}^{3}}
$$

Where $U_{3} \ldots$ voltage of the third harmonic frequency

(30 $\mathrm{kHz}$ ), $\mathrm{U}_{1} \ldots$ voltage of the basic frequency (10 kHz).

Values of THI after trimming have been standardized with respect to the resistance of the resistor before trimming with the use of the formula

$$
T H I_{R E}=\frac{R \cdot T H I_{T R M}}{R_{T R M}}
$$

Where $R$... resistance of the resistor before trimming, $R_{\text {TRIM }} \ldots$ resistance of the resistor after trimming, $\mathrm{THI} \mathrm{TRM}_{\mathrm{T}} \ldots$ index of nonlinearity before trimming, $\mathrm{THI}_{\mathrm{RE}} \ldots$ index of nonlinearity after trimming.

Trimming has been carried out through $80 \%$ of the width of the resistive path. Nonlinearity of the resistors trimmed by laser are shown in Fig. 4 and trimmed mechanically in Fig. 5.

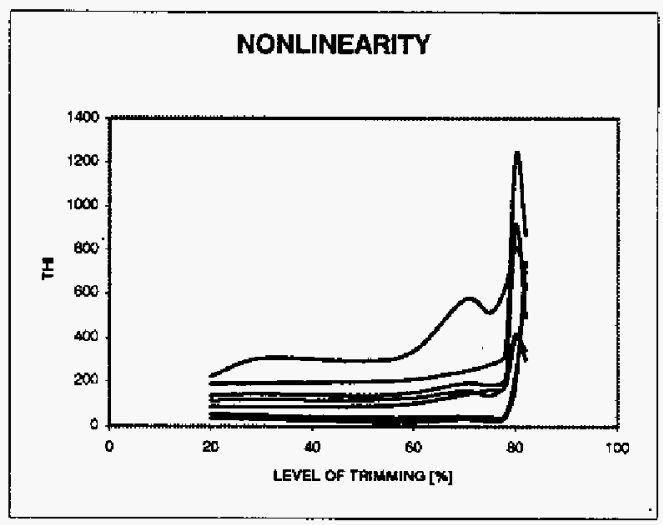

Fig. 4 Courses of nonlinearity of the trimmed resistors. Level of trimming has been from $20 \%$ to $80 \%$ of the width of the resistive layer. Trimming has been carried out by the AJ laser.

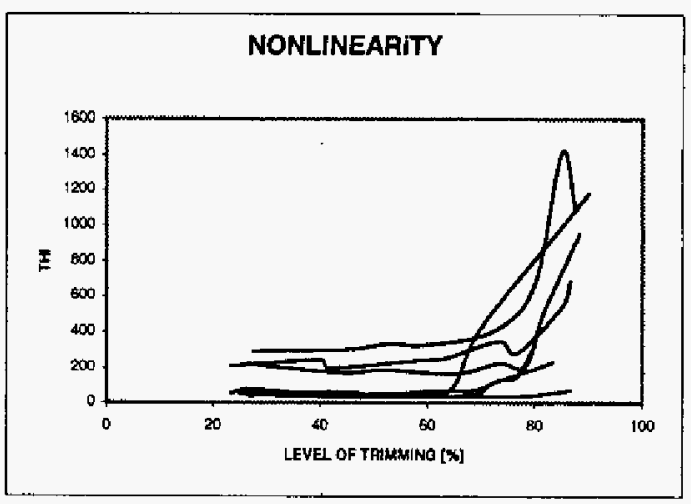

Fig. 5 Courses of nonlinearity of the trimmed resistors. Level of trimming has been from $20 \%$ to $80 \%$ of the width of the resistive layer. Trimming has been carried out mechanically.

Nonlinearity of non-trimmed and trimmed resistors has been compared. The values have been standardized with the use of the formula (5) before comparison. Typical diagrams have been obtained (Fig. 6 and Fig. 7). 


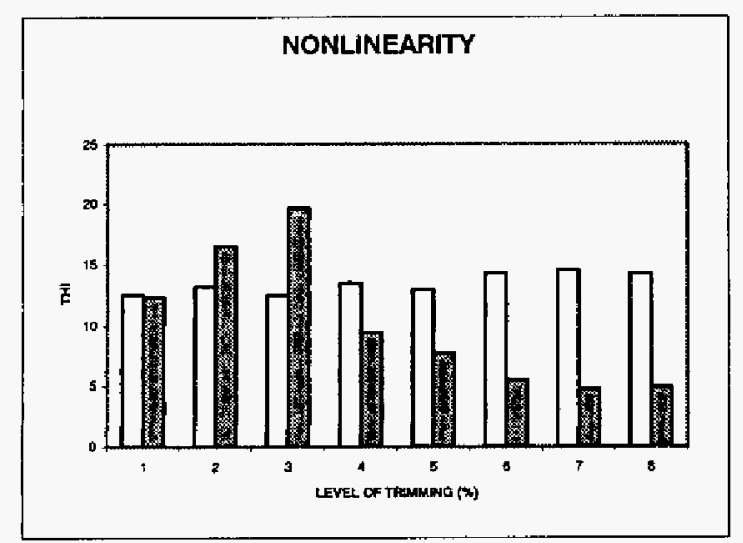

- Before trimming

- After trimming

Fig. 6 Comparison of nonlinearity of the resistors without trimming and the resistors trimmed by the laser

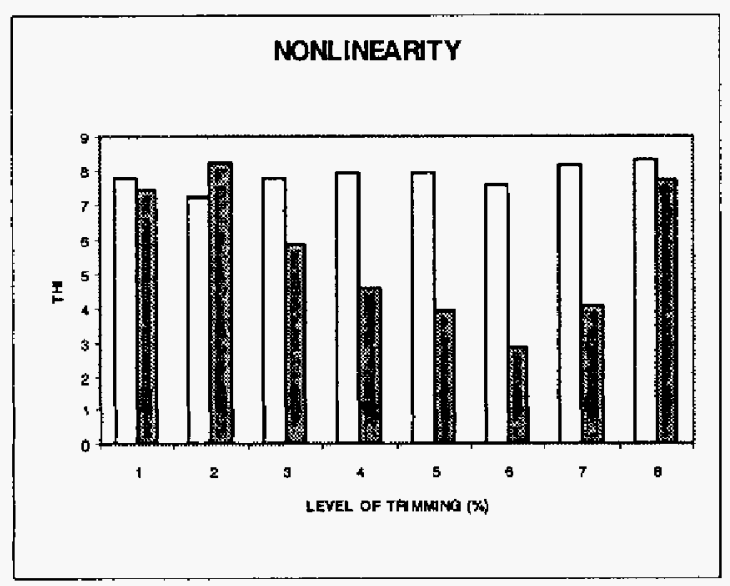

- Before trimning

- After trimming

Fig. 7 Comparison of nonlinearity of the resistors without trimming and the resistors trimmed mechanically

\section{Discussion and conclusions}

It has been shown that trimming has an influence on nonlinearity of thick film resistors. A simple theoretical analysis has shown that nonlinearity can be closely joined with the properties of the border of trimming groove.

The results of our experiments have shown that there are not significant differences between nonlinearity of the resistors trimmed by the laser or trimmed mechanically. The results have also shown that nonlinearity of the border of the trimming grove is not higher than nonlinearity of the basic resistive layer. This information is new and surprising, because it has been assumed that the nonlinearity of the resistors will increase after trimming. Such the situation has been found for low levels of trimming only in both types of trimming.

According to the Fig 4 and Fig. 5 it has been assumed that nonlinearity will increase after trimming and that the higher will be the level of trimming the higher will be nonlinearity. However, it is necessary to accept the fact that the resistance increases after trimming. Therefore it is necessary to re-calculate the nonlinearity for the same value of the resistance before mutual comparison (see Fig. 6 and Fig. 7).

It seems that the border of the trimming groove, where the layer has been melted and repeatedly solidificated, has higher resistivity than the basic layer and therefore its contribution to the final nonlinearity is lower than it has been assumed.

On the other hand the reason of lower nonlinearity can be caused also by the fact that the measurements have been carried out by the constant current density. For the resistors without trimming the total current has been given by this density multiplicated by the cross section of the layer. For trimmed resistors is the total current calculated as the product of the current density and the difference of the full cross section of the layer decreased by the cross section of the trimming groove. Therefore the cross section is lower and the total current is also lower. Therefore the nonlinear voltage generated by nonlinearity of contacts, which is for total nonlinearity of the resistor significant, decreases and the total nonlinearity of the resistor too.

\section{References}

1. Pelikanova, I.: Influence of the shape of thick film resistors on their nonlinearity. Ph.D. thesis. TU Prague. 2003.

2. Mach, P.:Properties of Adhesive Bonds Exposed to the Static and Dynamic Mechanical Load. Proc. ISSE 03. TU Kosice. Slovak Republic. 2003. 